\title{
ON THE RADICAL OF A GENERAL RING
}

\section{JAKOB LEVITZKI}

1. Introduction. The radical of a ring has been hitherto defined by using either the notion of a nilpotent or a nil-ideal. In the following we shall ascribe the term specialized radical to the sum $N_{\sigma}$ of all twosided nilpotent ideals of a ring $S$, and the term generalized radical to the sum $N_{\gamma}$ of all two-sided nil-ideals of the ring. In $\$ 2$ of the present note the notions of a semi-nilpotent ideal and its counterpart the semiregular ideal are introduced, and the term radical is suggested for the sum $N$ of all two-sided semi-nilpotent ideals of the ring. These notations may be justified by the following considerations:

(a) Each nilpotent ideal is semi-nilpotent, and each semi-nilpotent ideal is a nil-ideal.

(b) The radical $N$ is a two-sided semi-nilpotent ideal which contains also all one-sided semi-nilpotent ideals of the ring.

(c) The radical of $S / N$ is zero.

(d) The radical $N$ contains the specialized radical $N_{\sigma}$ and is a subset of the generalized radical $N_{\gamma}$.

(e) In the case of an algebra the notions: nilpotent, semi-nilpotent and nil-ideal are identical, and $N_{\sigma}=N=N_{\gamma}$; but if one turns to general rings, and replaces radical $N$ and semi-nilpotent ideals either by specialized radical $N_{\sigma}$ and nilpotent ideals or generalized radical $N_{\gamma}$ and nil-ideals, then some restriction has to be imposed on the ring $S$ in order to assure the validity of (b) and (c). ${ }^{1}$

These results are applied in $\$ 3$ to semi-primary rings (which will be called in short: $A$-rings).

2. The radical of a general ring. In this section certain theorems related to the radical of a general ring are proved.

Notation. If $r_{1}, \cdots, r_{n}$ is a finite set of elements in the ring $S$, then the ring generated by the $r$ will be denoted by $\left\{r_{1}, \cdots, r_{n}\right\}$.

Definition. $A$ right ideal is called semi-nilpotent if each ring gener-

\footnotetext{
Presented to the Society, October 31, 1942; received by the editors October 7, 1942.

1 Thus the specialized radical $N_{\sigma}$ is nilpotent (and hence the specialized radical of $S / N_{\sigma}$ is zero) if (as is well known) the maximal condition or (as can be shown) the minimal condition is satisfied by the two-sided ideals of the ring. As to the generalized radical $N_{\gamma}$, it has been proved (G. Koethe, Die Struktur der Ringe, Math. Zeit. vol. 32 (1930) pp. 161-186) that if each regular right ideal of $S$ contains a minimal regular right ideal, then $N_{\gamma}$ contains also all one-sided nil-ideals of the ring.
} 
ated by a finite set of elements belonging to the ideal is nilpotent. A right ideal which is not semi-nilpotent, is called semi-regular.

REMARKs. Each regular right ideal (that is, an ideal which contains elements which are not nilpotent) is evidently semi-regular, and each semi-regular right ideal is potent (that is, not nilpotent). Accordingly, each nilpotent right ideal is semi-nilpotent, and each semi-nilpotent right ideal is a right nil-ideal. One can easily find examples of rings containing semi-nilpotent right ideals which are not nilpotent. The problem of constructing a ring with right nil-ideals which are not semi-nilpotent, is equivalent to the construction of a potent nil-ring with a finite number of generators. It can be easily shown that such rings can always be found among the subrings of a ring with a generalized radical $N_{\gamma}$ (see $\$ 1$ ) which does not contain all one-sided nilideals of the ring.

THEOREM 1. The sum $R=\left(R_{1}, R_{2}\right)$ of two semi-nilpotent right ideals $R_{1}$ and $R_{2}$ is a semi-nilpotent right ideal.

Proof. Suppose $R$ is semi-regular, that is, $R$ contains a finite set $r_{01}, \cdots, r_{0 n}$ so that the ring $S^{*}=\left\{r_{01}, \cdots, r_{0 n}\right\}$ is potent. Write $r_{0 i}=r_{\sigma i}+r_{\gamma i}, \quad$ where $r_{\sigma i} \in R_{1}, \quad r_{\gamma i} \in R_{2}$, then evidently also $S^{* *}=\left\{r_{\sigma 1}, \cdots, r_{\sigma n}, r_{\gamma 1}, \cdots, r_{\gamma n}\right\}$ is potent, since $S^{*} \subseteq S^{* *}$. Define a set $r_{1}, \cdots, r_{m}$ as follows: (1) each $r_{i}$ is either an $r_{\sigma k}$ or an $r_{\gamma j}$, (2) the ring $\left\{r_{1}, \cdots, r_{m}\right\}$ is potent, (3) each ring generated by a proper subset of the $r_{i}$ is nilpotent. One obtains such a set by suppressing one way or another the greatest possible number of elements of the set $r_{\sigma 1}, \cdots, r_{\sigma n}, \cdots, r_{\gamma n}$, so that the remaining set still generates a potent ring. The set of the $r_{i}$ necessarily contains certain $r_{\sigma i}$ as well as certain $r_{\gamma k}$ (since the $r_{\sigma i}$ as well as the $r_{\gamma k}$ taken separately generate nilpotent rings), hence $m \geqq 2$. Now consider the rings $T=\left\{r_{1}, r_{2}, \cdots, r_{m}\right\}$ and $U=\left\{r_{2}, \cdots, r_{m}\right\}$. By the definition of the $r_{i}$ it follows that $T$ is potent, while $U$ is nilpotent. Denote by $\rho$ the index of the nilpotent element $r_{1}$ and by $\sigma$ the index of the nilpotent ring $U$. Denote by $w_{1}, \cdots, w_{s}$ the finite set of all elements of the form $r_{1}^{\lambda} r_{i_{1}} \cdots r_{i_{t}}$, where $0<\lambda<\rho ; 0<t<\sigma ; i_{j} \neq 1, j=1, \cdots, t$. Now, since $T$ is potent, it follows that for each positive integer $x$, elements $v_{1}, \cdots, v_{x}$ can be found so that each $v_{i}$ is a certain $r_{k}$, and the product $v_{1} \cdot v_{2} \cdots v_{x}$ is different from zero. From the definition of $\rho$ and $\sigma$ it follows that if $x>\rho, x>\sigma$, then the set $v_{1}, \cdots, v_{x}$ necessarily contains the element $r_{1}$ as well as elements different from $r_{1}$. Hence, by choosing an arbitrary integer $y$, and fixing $x$ so that $x>(\rho+\sigma)(y+2)$, we have $v_{1} \cdots v_{x}=f \cdot g_{1} \cdots g_{y} h$, where $f$ and $h$ are certain elements of $T$, while 
the $g_{i}$ are elements of the set $w_{1}, \cdots, w_{s}$. Since $g_{1} \cdots g_{y} \neq 0$, it follows that the ring $\left\{w_{1}, \cdots, w_{s}\right\}$ is potent. Since either $r_{1} \in R_{1}$ or $r_{1} \in R_{2}$, it follows that all the $w_{i}$ are either in $R_{1}$ or in $R_{2}$, which is a contradiction to the assumption that $R_{1}$ as well as $R_{2}$ are both semi-nilpotent.

REMARK. Theorem 1 probably does not hold if semi-nilpotent ideals are replaced by nil-ideals.

ThEOREM 2. The sum $N$ of all semi-nilpotent right ideals is a seminilpotent two-sided ideal, which contains also all semi-nilpotent left ideals of the ring.

Proof. First note, that by Theorem 1 it follows easily (by inducduction) that also the sum of any finite number of semi-nilpotent right ideals is again a semi-nilpotent right ideal. If now $r_{1}, \cdots, r_{n}$ is any finite set in $N$, then for each $r_{i}$ a finite number of semi-nilpotent right ideals $R_{1}^{i}, R_{2}^{i}, \cdots, R_{t_{i}}^{i}$ can be found so that $r_{i} \in\left(R_{1}^{i}, R_{2}^{i}, \cdots, R_{t_{i}}^{i}\right)$ $i=1, \cdots, n$. Hence the sum $R$ of all the $R_{k}^{i}$, which is a semi-nilpotent right ideal, contains all the $r_{i}$, which implies that the ring $\left\{r_{1}, \cdots, r_{n}\right\}$ is nilpotent. Since this is true for each set $r_{1}, \cdots, r_{n}$, it follows that $N$ is a semi-nilpotent right ideal. To prove that $N$ is a left ideal, and hence a two-sided semi-nilpotent ideal, we show that if $a \in N, s \in S$ and $R=(s a, s a S)$ then $R \subseteq N$. Indeed, suppose $R$ is semi-regular, that is, elements $r_{1}, \cdots, r_{n}$ of $R$ can be found so that the ring $\left\{r_{1}, \cdots, r_{n}\right\}$ is potent. Each $r_{i}$ has the form $r_{i}=s a s_{i}$, where $s_{i}$ is either an integer or an element of $S$. For an arbitrary positive integer $x$ we can find an element different from zero which has the form $s a s_{i_{1}} \cdots s a s_{i_{x}}$. Hence it follows that the ring $\left\{a s_{1} s, a s_{2} s, \cdots, a s_{n} s\right\}$ is potent, which is a contradiction, since $a s_{i} s \in N$. The remaining part of the theorem follows from the fact that (as can be similarly proved) also the sum of all semi-nilpotent left ideals is a semi-nilpotent two-sided ideal.

REMARK. Theorem 2 does not hold if semi-nilpotent ideals are replaced by nilpotent ideals and probably is not true if they are replaced by nil-ideals.

Definition of THE RAdical. The sum $N$ of all two-sided seminilpotent ideals of the ring (which by Theorem 2 contains also all onesided semi-nilpotent ideals) is called the radical of the ring.

THEOREM 3. If $N$ is the radical of $S$, then the radical of $S / N$ is zero.

Proof. We show that if $R[\geqq N]$ is a semi-regular right ideal in $S$, then $R / N$ is semi-regular in $S / N$. Indeed, let the elements $r_{1}, \cdots, r_{n}$ of $R$ generate the potent ring $T=\left\{r_{1}, \cdots, r_{n}\right\}$. If now $R / N$ is seminilpotent, then the ring $\bar{T}=(T+N) / N$ is nilpotent, that is, for a certain $\lambda$ we have $\bar{T}^{\lambda}=\bar{O}$ (where $\bar{O}$ is the zero of $S / N$ ), or $T^{\lambda} \subseteq N$. We 
denote by $u_{1}, \cdots, u_{m}$ the finite set of all products of the form $r_{i_{1}} \cdot r_{i_{2}} \cdots r_{i \lambda}$, and put $U=\left\{u_{1}, \cdots, u_{m}\right\}$. Since with $T$ also $T^{\lambda}$ for each $\lambda$ is potent, and since evidently $T^{\sigma \lambda+1} \subseteq U^{\sigma} T$, we have $U^{\sigma} \neq 0$ for each $\sigma$. On the other hand, by the definition of $U$, we have $U \subseteq T^{\lambda}$; hence $U \subseteq N$, which is a contradiction, since $N$ is semi-nilpotent.

As immediate consequences of the foregoing theorems we have these following theorems.

THEOREM 4. If in a ring $S$ each right ideal (other than zero) is semiregular, then also each left ideal (other than zero) is semi-regular.

THEOREM 5. An element s of a ring $S$ belongs to the radical $N$ of $S$ if, and only if, $s S \subseteq N$.

3. Remarks on $A$-rings. Using the notions of specialized radical, radical and generalized radical, one may define the following three classes of $A$-rings (that is, rings which have a similar structure as the algebras):

Definition. The ring $S$ is called a specialized $A$-ring, if the specialized radical $N_{\sigma}$ of $S$ is nilpotent, and the ring $S / N_{\sigma}$ is semi-simple.

Definition. The ring $S$ is called an $A$-ring, if the ring $S / N$ is semisimple. Herein $N$ denotes the radical of $S$.

Definition. The ring $S$ is called a generalized $A$-ring, if the ring $S / N_{\gamma}$ is semi-simple. Herein $N_{\gamma}$ denotes the generalized radical of $S$.

Using a well known theorem of $E$. Noether and the evident fact that the specialized radical of a ring is zero if the radical or the generalized radical of the ring is zero, we obtain the following characterization of $A$-rings and generalized $A$-rings :

Theorem 6. $A$ ring $S$ is an $A$-ring (generalized $A$-ring) if, and only if, the minimal condition is satisfied by the right ideals which contain the radical $N$ (generalized radical $N_{\gamma}$ ).

REMARKs. The condition of Theorem 6 concerning generalized $A$-rings, and "condition I" formulated by Koethe ${ }^{1}$ are of course equivalent. As to the specialized $A$-rings, since the discovery of E. Artin that rings with a maximal and minimal condition for the right ideals are specialized $A$-rings, various attempts have been made to generalize this result. However, a criterion for specialized $A$-rings along the line of Theorem 6 has not been found as yet.

Definition. $A$ ring $P$ with a unit is called a completely primary ring, if each right ideal of $P$ other than $P$ is semi-nilpotent. 
REMARK. Replacing semi-nilpotent ideals by nilpotent(nil-)ideals, one obtains a specialized completely primary (generalized completely primary) ring.

Definition. $A$ ring $Q$ with a unit is called a primary ring, if each two-sided ideal of $Q$ other than $Q$ is semi-nilpotent.

REMARK. Replacing semi-nilpotent ideals by nilpotent(nil-)ideals one obtains a specialized primary (generalized primary) ring.

THEOREM 7. A primary A-ring is isomorphic to a total matric-ring of a finite degree over a completely primary ring. Conversely, the total matric-ring of a finite degree $n$ over a completely primary ring $P$ is a primary A-ring.

Proof. The proof of the first part of the theorem can be derived by well known methods and may be omitted. The second part follows by known argument if only the following lemma is established: If in the matrix $\left(p_{i k}\right), i, k=1, \cdots, n$, the elements $p_{i k}$ are contained in the radical $N^{*}$ of $P$, then the matrix $\alpha=\left(p_{i k}\right)$ belongs to the radical $N$ of $Q$. To prove the lemma, suppose $\alpha$ is not in $N$, then by Theorem 5 we can find matrices $\alpha_{1}=\left(p_{i k}^{1}\right), \alpha_{2}=\left(p_{i k}^{2}\right), \cdots, \alpha_{t}=\left(p_{i k}^{t}\right)$ so that the subring $A=\left\{\alpha \alpha_{1}, \alpha \alpha_{2}, \cdots, \alpha \alpha_{t}\right\}$ of $Q$ is potent. Evidently, the subring $A^{*}=\left\{\cdots, p_{i k} p_{j l}^{r}, \cdots\right\}$ (where $i, k, j, l=1, \cdots, n ; r=1, \cdots, t$ ) of $P$ is then also potent. Since $p_{i k} \in N^{*}$, also $p_{i k} p_{j l}^{r} \in N^{*}$ which is a. contradiction, since $N^{*}$ is semi-nilpotent.

REMARK. The second part of Theorem 7 is a generalization of an analogous theorem concerning specialized completely primary rings, since one can easily find examples of completely primary rings which are not specialized completely primary rings. It does not seem probable that an analogous theorem could be proved for generalized completely primary rings. This has been attempted by Koethe under the assumption that the generalized completely primary ring $P$ satisfies the minimal condition for the right ideals; however, this condition implies that $P$ is a specialized completely primary ring.

HEBREW UNIVERSITY

Jerusalem, Palestine 